\title{
Investigation of UV Matrix-Assisted Laser Desorption Fourier Transform Mass Spectrometry for Peptides
}

\author{
R. L. Hettich and M. V. Buchanan \\ Analytical Chemistry Division, Oak Ridge National Laboratory, Oak Ridge, Tennessee, USA
}

\begin{abstract}
Ultraviolet matrix-assisted laser desorption can be used to enhance formation of $[\mathrm{M}+\mathrm{H}]^{+}$, $[\mathrm{M}+\mathrm{Na}]^{+}$, and $\left[\mathrm{M}+\mathrm{K}^{+}\right.$ions from small peptides for Fourier transform mass spectrometry (FTMS). In accord with laser desorption (LD) time-of-flight experiments, matrices such as nicotinic acid and 2-pyrazinecarboxylic acid exhibit strong enhancement effects (i.e., formation of abundant protonated and cationized molecules for the analyte with virtually no fragment ions) for $266 \mathrm{~nm}$ LD/FTMS, whereas pyrazinedicarboxylic acid provides no matrix enhancement at this wavelength. Both sinapinic acid and coumarin-120 provide strong matrix enhancement effects for the 355-nm LD of peptides. For the small peptides examined in this study, no significant differences in the abundance of fragment ions were observed between the 266- and 355-nm wavelengths. Matrix-assisted LD/FTMS is useful for the generation and characterization of ions corresponding to protonated and cationized molecules from virtually all biological compounds with molecular weights up to 2000. The lack of observation of biological ions with $\mathrm{m} / z>2500$ may be related to inefficient trapping of these laser-desorbed ions or instrumental detection limitations of FTMS and is under further investigation. (I Am Soc Mass Spectrom 1991, 2, 22-28)
\end{abstract}

$\mathrm{L}$ aser desorption (LD) mass spectrometry has been used extensively for the ionization and Istructural characterization of nonvolatile, thermally labile compounds. The recent development of matrix-assisted UV LD techniques has made possible the generation of ions at $m / z>50,000$ for extremely large compounds $[1,2]$. This technique along with the electrospray ionization (ESI) techniques [3-5] has sparked a renewed interest in the mass spectral examination of large biomolecules such as proteins and oligonucleotides.

Matrix-assisted LD uses a sample prepared by mixing a small quantity of analyte such as a peptide with a large excess of matrix that helps absorb and dissipate the laser radiation, thereby reducing fragmentation of the analyte compound. A variety of laser wavelengths and sample matrices have been examined with time-of-flight (TOF) mass spectrometry [2, 6]. The resulting mass spectra usually contain protonated or deprotonated molecules of the analyte with virtually no fragment ions. The low-mass ions from the matrix are usually deflected to prevent them from reaching the detector and generating a high background in the low-mass region [6]. Although this ionization technique has been developed and used primarily with TOF instruments, research is currently

Address reprint requests to Robert L. Hettich, Analytical Chemistry Division, Oak Ridge National Laboratory. Oak Ridge, TN 37831-6120. in progress to demonstrate this matrix-assisted technique for trapped ion instruments, such as three-dimensional quadrupole ion traps and Fourier transform ion cyclotron resunance mass spectrometers (FTMS). The capabilities of FTMS for wide mass range, high-resolution measurements and ion-trapping experiments (for examining tandem mass spectrometry and ion-molecule reactions) suggest that this instrumental technique would be useful for the detailed structural characterization of large ions generated by the matrix-assisted ionization method. The lack of success to date in matrix-assisted enhancement for LD/FTMS experiments for any biological compounds has implied that there may be inherent FTMS instrumental limitations that prohibit coupling this mass spectral technique with the matrix-assisted ionization process. The objective of this report is to demonstrate that matrix-assisted LD, with either 266- or 355-nm radiation, can be successfully used with FTMS for the formation of abundant protonated or cationized molecules for small peptides.

\section{Experimental}

All experiments were performed with an Extrel FTMS-2000 Fourier transform mass spectrometer (3Tesla magnet) (Madison, WI) equipped with a Quanta Ray DCR-11 Nd:YAG pulsed laser [7]. The laser outputs a donut-shaped beam with a pulse length of 5-8 
ns and was operated in the $Q$-switched mode to generate radiation at 1064,355 , and $266 \mathrm{~nm}$. The maximum laser energies available from the Nd:YAG laser (at $10 \mathrm{~Hz}$ operation) were $300 \mathrm{~mJ} /$ pulse at 1064 $\mathrm{nm}, 35 \mathrm{~mJ} /$ pulse at $355 \mathrm{~nm}$, and $20 \mathrm{~mJ} /$ pulse at 266 $\mathrm{nm}$ and were optimized daily with a laser power meter. A periscope assembly (with two fused silica right-angle prisms) and a sapphire vacuum window were used to direct the laser beam into the vacuum system of the FTMS. An off-axis aluminium parabolic mirror, mounted inside the vacuum system, was used to focus the laser radiation onto the tip of the solids probe. Although this mirror is made for use with IR laser radiation, it also was found to be acceptable for focusing UV radiation as well, although some distortion of the spot occurs. The laser spot size was measured by examining with a microscope the burn marks made on thermal paper by laser impact after the aluminum focusing mirror. For $266 \mathrm{~nm}$, the spot size was found to be approximately $250 \mu \mathrm{m} \times 250 \mu \mathrm{m}$. The power density at the probe tip was not directly measured but was estimated to be in the low $10^{6}$ $W / \mathrm{cm}^{2}$ range for $266 \mathrm{~nm}$ on the basis of knowledge of the laser pulse energy, transmission optics, and the spot size. Increasing the laser power density above this value resulted in increased fragmentation of the analyte, in accord with matrix-assisted LD results observed by other laboratories [6].

Briefly, the experiment consisted of the following sequence. The laser was triggered from the Extrel FTMS computer at the beam event to ionize the sample. The trapping plates of the FTMS cell were held at $+2 \mathrm{~V}$ during the entire experimental sequence. Following the laser pulse, an ejection pulse was used to eliminate the $[\mathrm{M}+\mathrm{H}]^{+}$ions of the matrix compound (i.e., $m / z 124$ for nicotinic acid). The duration of this ejection pulse was $150 \mathrm{~ms}$ (the exact delay time did not significantly affect the spectrum) and was immediately followed by broadband excitation (2.0 $\mathrm{MHz}$ bandwidth at a sweep rate of $2.0 \mathrm{kHz}$ ) and detection under medium resolution conditions $(32 \mathrm{~K}-64 \mathrm{~K}$ data points). All spectra shown in this report were obtained by one laser pulse and have resolutions of 200-1000 (FWHM). Higher resolution spectra (1000-2000 FWHM) were obtained by trapping the ions for several seconds after the laser pulse to allow the neutral species to pump away before ion detection. A complete mass spectrum for either positive or negative ions was obtained for each laser shot, although signal averaging could be used to improve the spectra. Several laser shots could be taken at the same position before the probe had to be rotated for fresh sample. Even though optimum performance of the YAG laser is obtained when operating at $10 \mathrm{~Hz}$, furing the laser only once at the beam event still provided sufficient laser energy for the matrix-assisted LD experiments. Clearly, the shot-to-shot reproducibility under single-shot conditions varies, which affects the relative intensities of the ions. However, signal-aver-
Table 1. Peptides examined by LD/FTMS

\begin{tabular}{lc}
\hline Peptide & Mol wt \\
\hline \hline Leucine enkephalin & 555.6 \\
Methionine enkephalin & 573.5 \\
Gramicidin S & 1141.5 \\
Substance P & 1347.7 \\
Gramicidin D & 1882 \\
Bovine insulin A-chain & 2535 \\
Bovine insulin & 5733.5 \\
\hline
\end{tabular}

aging multiple laser shots by using the experimental sequence outlined above allows the laser to operate at approximately $5 \mathrm{~Hz}$ and tends to reduce the fluctuations in ion abundances

The sample was prepared by mixing $1 \mu \mathrm{L}$ of an aqueous analyte solution (approximately $10^{-4} \mathrm{M}$ ) with a few microliters of an aqueous matrix solution (approximately $10^{-2} \mathrm{M}$ ), based on sample concentrations reported for the TOF experiments $[2,6]$. This solution was then placed on the tip of a stainless steel solids probe, which was inserted into the vacuum system of the FTMS. The pressure in the vacuum system of the FTMS was allowed to reach at least $2 \times 10^{-7}$ torr or lower before spectral acquisition (this typically required less than $15 \mathrm{~min}$ for the nicotinic acid samples). Even though the nicotinic acid matrix is volatile, the LD experiments could be performed for at least 30 min before the abundance of fragment inns increased substantially, indicating loss of the matrix enhancement effects. For these initial investigations, extensive examination of detection limits was not determined. Typically, the amount of analyte placed on the probe was in the range of $10-100$ pmol, although this amount certainly exceeded that required for the LD process.

\section{Results and Discussion}

\section{Laser Desorption Without Matrices}

A variety of experimental conditions were examined for small peptides in order to probe the nature of the matrix-assisted LD for application to FTMS. Initial experiments were conducted by depositing the peptides directly onto the stainless steel surface of the solids probe (without a matrix). Reference spectra were obtained with 1064- and 266-nm LD and were used to judge the enhancement of the matrix-assisted LD experiments. Table 1 lists the peptides that were examined in this study. Laser desorption spectra, obtained with either 1064- or 266-nm radiation, of methionine enkephalin (MW 573.5) and leucine enkephalin (MW 555.6) deposited directly on the metal substrate yielded abundant $[\mathrm{M}+\mathrm{H}]^{+},[\mathrm{M}+\mathrm{Na}]^{+}$, and $[\mathrm{M}+\mathrm{K}]^{+}$ions. The negative-ion spectra of the enkephalins exhibited $[\mathrm{M}-\mathrm{H}]^{-}$ions as well as fragment ions that could be used to deduce their amino acid sequences. The spectra for these two compounds were wavelength-independent under these experimental conditions. Results by Chiarelli and Gross [8] 
Table 2. Effect of laser wavelength and matrix composition on laser desorption ${ }^{3}$

\begin{tabular}{lcccccccc}
\hline & $1064 \mathrm{~nm}$ & \multicolumn{3}{c}{$266 \mathrm{~nm}$} & & \multicolumn{2}{c}{$355 \mathrm{~nm}$} \\
\cline { 5 - 7 } \cline { 5 - 7 } & SS & SS & NA & PA & PDA & & SA & C120 \\
\hline \hline Leucine enkephalin & + & + & + & + & + & & + & + \\
Methionine enkephalin & + & + & + & + & + & + & + \\
Gramicidin S & + & + & + & + & - & + & + \\
Substance P & - & - & + & + & - & + & + \\
Gramicidin D & - & - & + & + & - & + & + \\
Bovine insulin A-chain & - & - & - & - & - & - & - \\
Bovine insulin & - & - & - & - & - & - & - \\
\hline
\end{tabular}

Abbreviations: C120, coumarin 120 ; NA, nicotinic acid; PA, pyrazinecarboxylic acid PDA, pyrazinedicarboxylic acid; SA, sinapinic acid; SS, stainless steel with no matrix. $a^{+},[\mathrm{M}+\mathrm{H}]^{+},[\mathrm{M}+\mathrm{Na}]^{+},[\mathrm{M}+\mathrm{K}]^{+}$ions were abserved; - , these ions were not observed.

indicated that the LD spectra of nucleosides deposited on copper substrates were virtually wavelength-independent, although there were slight differences in the positive-ion abundances for the pyrimidines. Gramicidin S (MW 1141.5) can also be ionized from stainless steel with either wavelength to give primarily $[M+$ $\mathrm{Na}]^{+}$. Because gramicidin $S$ is a cyclic decapeptide and can easily coordinate to metal cations such as $\mathrm{Na}^{+}$or $\mathrm{K}^{+}$, it can be desorbed more easily than linear peptides of similar size [9]. No characteristic negative ions were observed for gramicidin S. Peptides with $M W>1300$, such as substance $P$ and gramicidin $D$, did not reveal protonated or cationized molecules by LD from stainless steel with either wavelength. Other research groups have demonstrated that the addition of $\mathrm{KCl}$ to peptide samples prior to infrared LD often aids in the formation of $[\mathrm{M}+\mathrm{K}]^{+}$ions (for example, gramicidin D [10] and substance $P$ [9]), although the signal levels are quite low.

\section{6-nm Matrix-Assisted LD/FTMS}

Following protocols outlined by Karas and Hillenkamp [2], 266-nm LD/FTMS with nicotinic acid (3-pyridine carboxylic acid) as the matrix was examined. This matrix-assisted ionization process dramatically improved the abundance of the protonated $[\mathrm{M}+$ $\mathrm{H}^{+}$and cationized $[\mathrm{M}+\mathrm{Na}]^{+},[\mathrm{M}+\mathrm{K}]^{+}$ions. Table 2 summarizes the success of these FTMS experiments for peptides with various matrices and laser wavelengths. Even though $[\mathbf{M}+\mathbf{H}]^{+}$ions could be obtained from the enkephalins by LD from stainless steel without a matrix, the addition of nicotinic acid for their matrix significantly enhanced the abundance of protonated species while reducing the amount of fragmentation observed. The LD/FTMS spectra of substance $P$ (MW 1347.7) with and without nicotinic acid (all other experimental factors held constant) are shown in Figures 1 and 2 and illustrate the remarkable signal enhancement with nicotinic acid. The use of nicotinic acid as the matrix for 266-nm LD/FTMS enhances the formation of $[\mathrm{M}+\mathrm{H}]^{+},[\mathrm{M}+\mathrm{Na}]^{+}$, and $[\mathrm{M}+\mathrm{K}]^{+}$ions for peptides with molecular weights at least up to 2000, as shown in Table 2 . The negative-ion spectra of these peptides did not contain characteristic $[\mathrm{M}-\mathrm{H}]^{-}$or fragment ions. Initial investigations of peptides exceeding MW 2500, such as bovine insulin A-chain (MW 2535) and bovine insulin (MW 5733.5), failed to reveal characteristic ions with the 266$\mathrm{nm} /$ nicotinic acid conditions. The lack of success with these larger peptides may be due to either poor ion trapping in the FTMS cell or limitations in ion detection with conventional FTMS hardware. Spengler et al. [11] reported that ions generated by the matrix-assisted technique have significant kinetic energy distributions; ions with $m / z<4000$ have energies below 5 $\mathrm{eV}$, whereas ions with $m / z>4000$ may have energies exceeding $30 \mathrm{eV}$. Because FTMS experiments are quite sensitive to the kinetic energies of the ions, these larger ions would be very difficult to trap in the FTMS cell. Another possible limitation with these experiments is ion detection with conventional FTMS hardware. We have been successful in generating and detecting ions in the $m / z$ 2000-4000 range for polyethylene glycol polymers with our instrument, while other FTMS groups have reported detecting ions at $m / z>8000$ for synthetic polymers [12] and cesium iodide clusters [13]. However, several research groups have observed difficulty in generating and detecting ions at $m / z>3500$ for any biological compounds $[14,15]$. Although the reasons for these detection limitations for biological compounds are currently unknown, extensive research is under way in our laboratory as well as in many other FTMS laboratories to examine and alleviate these detection problems with larger magnets, modified cell designs, and tailored ion-manipulation techniques [15].

Beavis and Chait [6] examined a variety of matrices and determined that 2-pyrazinecarboxylic acid can also be used for 266-nm LD experiments [6]. This matrix works as well as nicotinic acid for the FTMS experiments, as shown in Table 2. Figure 3 illustrates the positive-ion spectrum for a mixture of gramicidin $S$ and $D$ with pyrazinecarboxylic acid as the matrix. Adduct ions of the analyte with the matrix were not observed for these compounds, although such ions have been observed for larger peptides in the TOF experiments [6]. This ionization enhancement is highly 


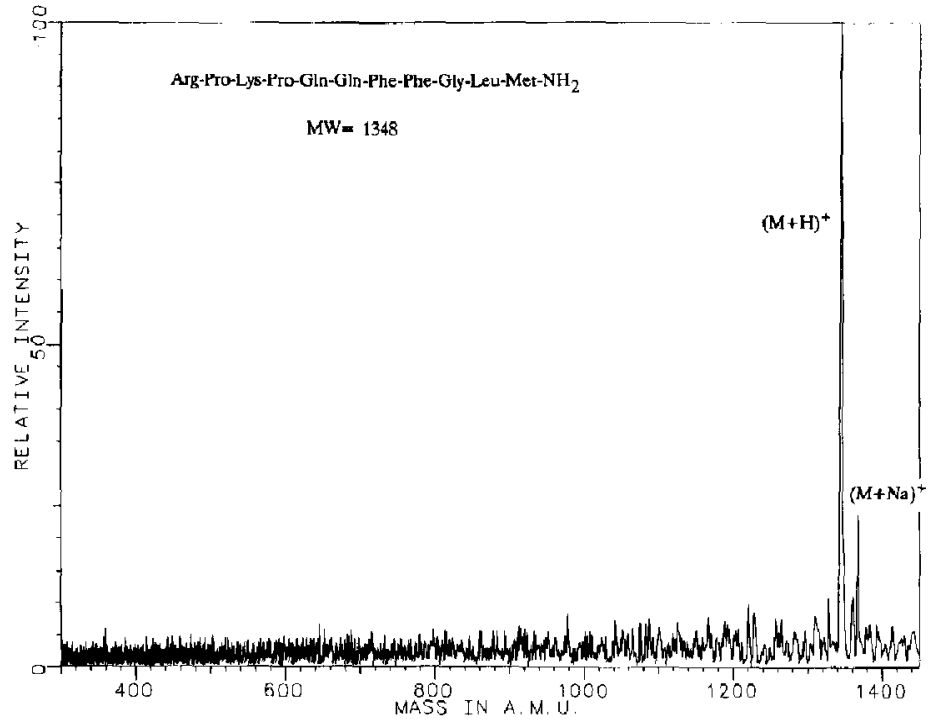

Figure 1. Matrix-assisted LD spectrum of substance $P$ (266 nm; nicotinic acid matrix). dependent on the chemical structure of the matrix compound. For example, pyridinecarboxylic acids provide matrix enhancement, whereas pyridinedicarboxylic acids do not exhibit this effect [6]. 2,3Pyrazinedicarboxylic acid is structurally similar to 2pyrazinecarboxylic acid but was found not to provide matrix enhancement for LD/FTMS experiments. Figure 4 shows the spectrum obtained for the gramicidin $\mathrm{S}$ and $\mathrm{D}$ mixture under conditions identical to those of Figure 3 except that pyrazinedicarboxylic acid was used as the matrix. Ions corresponding to the cationized molecules are not observed for either gramicidin, which verifies that the matrix selection has a large effect on the ionization conditions even at $m / z<$ 2500. This experiment provides further evidence for matrix-assisted enhancement as opposed to differences in sample preparation. As observed for the nicotinic acid FTMS experiments, peptide ions with $m / z>2500$ were not observed with pyrazinecarboxylic acid matrix.

\section{5-nm Matrix-Assisted LD/FTMS}

The goal of the matrix-assisted ionization technique is to pump the laser energy primarily into the matrix and not into the analyte [2]. Because many compounds absorb radiation at $266 \mathrm{~nm}$, LD at this wavelength excites both the matrix and the analyte, often resulting in fragmentation of the analyte molecules. To avoid this problem, an alternative wavelength and

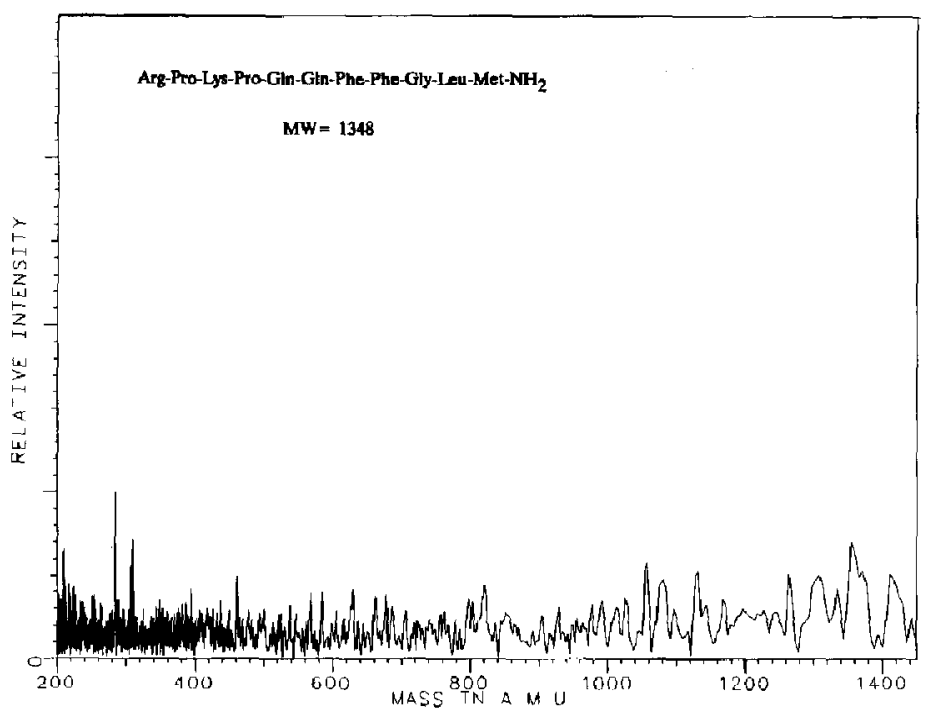

Figure 2. Laser desorption of substance P (266 $\mathrm{nm}$; conditions identical to those of Figure 1 except that no nicotinic acid was used). 
Figure 3. Matrix-assisted LD spectrum of gramicidin $S$ and gramicidin D mixture $(266 \mathrm{~nm}$ 2-pyrazinecarboxylic acid matrix).

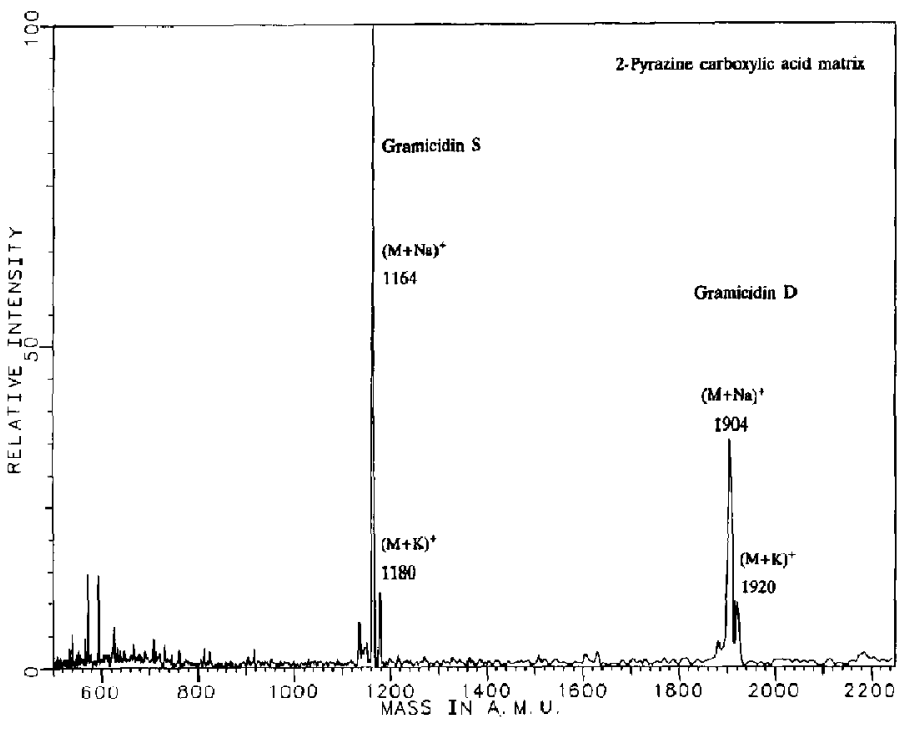

appropriate matrix can be chosen to minimize spectral absorption by the analyte. Beavis and Chait [16] suggested that sinapinic acid (3,5-dimethoxy-4-hydroxycinnamic acid) provides an excellent matrix for the 355-nm LD of peptides. Figure 5 illustrates the LD/FTMS spectrum for gramicidin D obtained with a sinapinic acid matrix and $355-\mathrm{nm}$ laser radiation. In this case, the laser power of the 355-nm line was adjusted to be approximately equal to that used for the 266-nm line to minimize differences due to laser power. Note the enhanced $[\mathrm{M}+\mathrm{H}]^{+} /\left[\mathrm{M}+\mathrm{Na}^{+}\right.$ratio for gramicidin D in Figure 5 relative to the same ratio in Figure 3. Although formation of $[\mathrm{M}+\mathrm{K}]^{+}$ ions was reported previously for gramicidin D [10],
Figure 5 reveals a relatively large abundance of $[\mathrm{M}+$ $\mathrm{H}^{+}$ions as well. Formation of abundant $[\mathrm{M}+\mathrm{H}]^{+}$ ions is desirable, because the fragmentation of these ions is often more structurally informative than the fragmentation of $[\mathrm{M}+\mathrm{K}]^{+}$ions $[9,14]$. Because most of the peptides examined in this study did not frag. ment significantly with either $266-\mathrm{nm}$ or $355-\mathrm{nm} \mathrm{LD}$ conditions, it was difficult to ascertain the difference between $355-\mathrm{nm}$ or $266-\mathrm{nm}$ LD. However, in general, better quality spectra (i.e., greater signal-to-noise ratios) were obtained for the $355-\mathrm{nm} \mathrm{LD}$. This wavelength difference should be more pronounced for compounds that absorb strongly at $266 \mathrm{~nm}$, such as oligonucleotides. Table 2 indicates that this matrix
Figure 4. Laser desorption spectrum of gramicidin $S$ and gramicidin D mixture $(266 \mathrm{~nm}$; 2,3-pyrazinedicarboxylic acid matrix). No matrix enhancement is observed in this case.

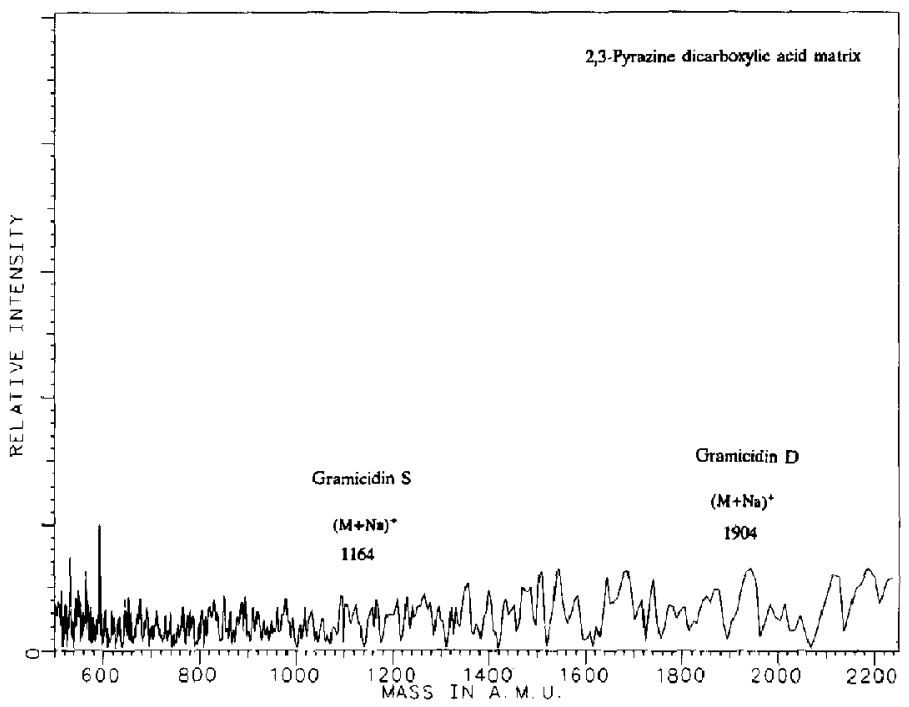




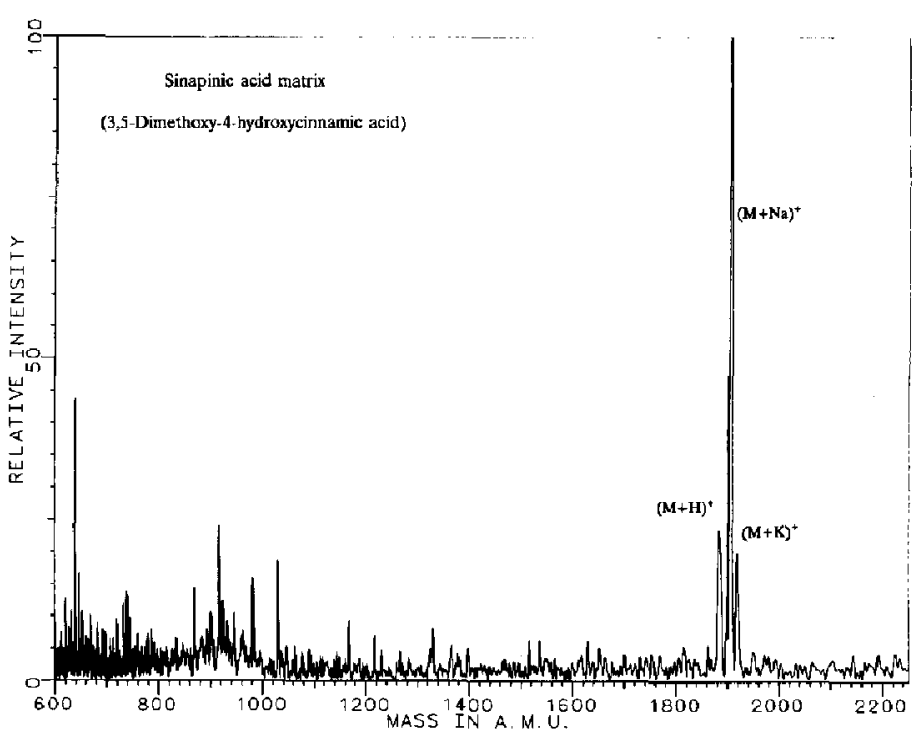

Figure 5. Matrix-assisted LD spectrum of gramicidin $\mathrm{D}$ (355 $\mathrm{nm}$; sinapinic acid matrix). also enhances formation of $[\mathrm{M}+\mathrm{H}]^{+},[\mathrm{M}+\mathrm{Na}]^{+}$, and $[\mathrm{M}+\mathrm{K}]^{+}$ions of peptides up to $m / z 2000$. Ions corresponding to cationized molecules for peptides with MW > 2500 were not observed for the 355-nm LD experiments, verifying the same limit observed for the $266-\mathrm{nm}$ results.

The function of the matrix to prevent fragmentation of the analyte molecule is not only due to its spectral absorption of the incident radiation but also includes other factors such as solvation of the analyte and matrix volatility $[2,6]$. To probe the spectral absorption effect of the matrix compound, we examined other compounds that absorb strongly at $355 \mathrm{~nm}$, such as the coumarin dyes, as matrices for peptides. The positive-ion LD of gramicidin $D$ with coumarin120 (7-amino-4-methylcoumarin) as the matrix and 355-nm laser radiation revealed abundant ions at [M $+[\mathrm{II}]^{+},[\mathrm{M}+\mathrm{Na}]^{+}$, and $[\mathrm{M}+\mathrm{K}]^{+}$. As indicated in Table 2, this matrix is as successful as sinapinic acid in enhancing the 355-nm LD of small peptides.

\section{Conclusions}

Ultraviolet matrix-assisted LD FTMS is useful for enhancing formation of protonated and cationized molecules for peptides with molecular weights less than 2500. The choice of matrices and wavelengths for the FTMS experiments agrees very well with results obtained for TOF experiments $[2,6]$ and verifies matrix-enhancement effects for LD/FTMS. Matrices such as nicotinic acid and pyrazinecarboxylic acid enhance formation of $[\mathrm{M}+\mathrm{H}]^{+},[\mathrm{M}+\mathrm{Na}]^{+}$, and $[\mathrm{M}+\mathrm{K}]^{+}$ ions for the 266-nm LD process, whereas pyrazinedicarboxylic acid does not provide matrix assistance at this wavelength. Sinapinic acid and coumarin-120 were both found to be strong matrices for the 355-nm LD of peptides. For the peptides examined in this study, no significant spectra differences were observed for 266-nm versus 355-nm LD. Matrix-assisted LD/FTMS is useful for generating characteristic ions that provide $\mathrm{MW}$ information (at the low picomole level) of biomolecules such as peptides and oligonucleotides with MWs up to at least 2000 . The lack of observation of peptide ions at $\mathrm{m} / z>2500$ may be due to inefficient trapping of the laser-desorbed ions or current FTMS detection limitations. Further research will be directed at examining the origin of this apparent FTMS limitation for the observation of large ions ( $m / z>3000$ ) from biological compounds. Methods to cool the ions generated by the matrix-assisted LD technique as well as better FTMS ion-detection capabilities will be investigated in detail for extending the mass range of FTMS for biological compounds.

\section{Acknowledgment}

This research was supported by Office of Health and Environmental Research, U.S. Department of Energy under contract DE-AC05-84OR21400 with Martin Marietta Energy Systems, Inc.

\section{References}

1. Tanaka, K.; Waki, H.; Ido, Y.; Akita, S.; Yoshida, Y.; Yoshida, T. Rapid Commun. Mass Spectrom. 1988, 2, 151.

2. Karas, M.; Hillenkamp, F. Anal. Chem. 1988, 60, 2299.

3. Yamashita, M.; Fenn, J. B. J. Phys. Chem. 1984, 88, 4451.

4. Olivares, J. A.; Nguyen, N. T.; Yonker, C. R.; Smith, R. D. Anal. Chem. 1987, 59, 1230.

5. Bruins, A. P.; Covey, T. R.; Henion, J. D. Anal. Chem. 1987, $59,2642$.

6. Beavis, R. C.; Chait, B. T. Rapid Commun. Mass Spectrom. 1989, 3, 233.

7. For a discussion of Fourier transform mass spectrometry (FTMS) and laser desorption FTMS, see Fourier Transform 
Mass Spectrometry; Evolution, Innozation, and Applications, ACS Symp. Ser. 359; Buchanan, M. V., Ed.; American Chemical Society: Washington, D.C., 1987.

8. Chiarelli, M. P.; Gross, M. L. J. Phys. Chem. 1989, 93, 3595.

9. Cody, R. B., Jr.; Kinsinger, J. A.; Ghaderi, S.; Amster, I. J.; McLafferty, F. W.; Brown, C. E. Anal. Chim. Acta. 1985, $178,43$.

10. Cody, R. B., Jr.; Amster, I. J.; McLafferty, F. W. Proc. Natl. Acad. Sci. U.S.A. 1985, 82, 6367.

11. Spengler, B.; Pan, Y.; Cotter, R. J., Matrix-assisted UV laser desorption of proteins and oligonucleotides on a WileyMcLaren time-of-flight mass spectrometer. Presented at the 38th ASMS Conference on Mass Spectrometry and Allied Topics, Tucson, June 3, 1990.
12. Ijames, C. F,; Wilkins, C. L. J. Am. Chem. Soc. 1988, 110, 2687.

13. Lebrilla, C. B.; Wang, D. T.-S.; Hunter, R. L.; McIver, R. L., Ir. Anal. Chem. 1990, 62, 878.

14. Hunt, D. F.; Shabanowitz, J.; Yates, J. R. III; Zhu, N.-Z.; Russell, D. H.; Castro, M. Proc. Natl. Acad. Sci. U.S.A. 1987, 84,620 .

15. For a review of the current status of FTMS research, see Wilkins, C. L.; Chowdhury, A. K.; Nuwaysir, L. M.; Coates, M. L. Mass Spectrom. Rev. 1989, 8, 67.

16. Beavis, R. C.; Chait, B. T., Laser desorption of proteins in unpurified biological fluids: a new tool for biological research. Presented at the 38 th ASMS Conference on Mass Spectrometry and Allied Topics, Tucson, June 3, 1990. 